\title{
Nomophobia Questionnaire: Propriedades Psicométricas para o Contexto Brasileiro
}

\section{Nomophobia Questionnaire: Psychometric Properties for the Brazilian Context}

\author{
Paulo Gregório Nascimento da Silva ${ }^{1}$, Mayara de Oliveira Silva Machado², \\ Andrezza Mangueira Estanislau ${ }^{3}$, Carlos Eduardo Pimentel $^{4}$, Patrícia Nunes da Fonsêca ${ }^{5}$ e \\ Emerson Diógenes de Medeiros ${ }^{6}$
}

\begin{abstract}
Resumo
A nomofobia é a ansiedade de separação do smartphone, que pode acarretar problemas psicossociais. A pesquisa buscou explorar os parâmetros psicométricos da Nomophobia Questionnaire (NMP-Q) no Brasil. Participaram 500 pessoas da população geral de diferentes estados brasileiros $\left(M_{\text {idade }}=27.84 ; D P_{\text {idade }}=8.69\right)$, em maioria mulheres $(67.4 \%)$, que foram divididos em dois estudos. No primeiro observaram-se evidências de validade fatorial e consistência interna para os 18 itens da NMP-Q. No segundo estudo foi corroborada a estrutura tetrafatorial (incapacidade de comunicação, perda de conexão, incapacidade de acessar informações e renúncia da conveniência), tendo indicadores de ajuste adequados (CFI=.94, TLI=.93, RMSEA=.08), apresentando coeficientes de fidedignidade aceitáveis, além de verificar validade convergente por meio das correlações entre os fatores da NMP-Q e vício no smartphone. Os resultados demonstram evidências de adequação psicométrica da NMP-Q, podendo ser uma ferramenta útil para compreender antecedentes e consequentes da nomofobia.
\end{abstract}

Palavras-chave: nomofobia, vício no smartphone, medida, validade, precisão

\begin{abstract}
Nomophobia is the separation anxiety from smartphone, which can lead to psychosocial problems. The study sought to explore the psychometric parameters of the Nomophobia Questionnaire (NMP-Q) in Brazil. 500 people from the general population of different Brazilian states $\left(M_{a g e}=27.84 ; S D_{a g e}=8.69\right)$, mostly women (67.4\%), who were divided into two studies. In the first one, evidence of factorial validity and internal consistency were observed for the 18 items of NMP-Q. In the second study, the tetrafatorial structure (inability to communicate, loss of connection, inability to access information and disclaimer of convenience) was corroborated, with adequate adjustment indicators (CFI=.94, TLI=.93, RMSEA=.08), presenting acceptable reliability coefficients, as well as verifying convergent validity through correlations between NMP-Q factors and smartphone addiction. The results demonstrate evidence of the psychometric adequacy of NMP-Q, which may be a useful tool to understand antecedents and consequents of nomophobia.
\end{abstract}

Keywords: nomophobia, smartphone addiction, measure, validity, precision

O presente artigo contou com apoio do CNPq por meio da bolsa de produtividade a quinta autora, da UFPI pela concessão de bolsa de produtividade de Pesquisa ao sexto autor e da CAPES, que concedeu bolsas de doutorado ao primeiro autor e de mestrado as segunda e terceira autoras. Aproveitamos para demonstrar nossa gratidão a essas instituições. Toda correspondência deverá ser encaminhada à Paulo Gregório Nascimento da Silva, telefone: (86) 999676859. Email: silvapgn@gmail.com; Universidade Federal da Paraíba. Castelo Branco, João Pessoa/PB. CEP: 58059-900. Centro de Educação, Departamento de Psicopedagogia.

\footnotetext{
${ }^{1}$ Mestre em Psicologia e Doutorando em Psicologia Social pela Universidade Federal da Paraíba - UFPB, João Pessoa-PB, Brasil. Tel.: +55(86)99967-6859. E-mail: silvapgn@gmail.com.

2 Psicopedagoga e Mestranda em Psicologia Social pela Universidade Federal da Paraíba - UFPB, João Pessoa-PB, Brasil. E-mail: mayara_machado94@hotmail.com

3 Psicopedagoga e Mestranda em Psicologia Social pela Universidade Federal da Paraíba - UFPB, Brasil. E-mail: andrezza.estanislau@hotmail.com

${ }^{4}$ Doutor em Psicologia Social, do Trabalho e das Organizações (PSTO). Professor Adjunto III do Departamento de Psicologia e da PósGraduação em Psicologia Social da Universidade Federal da Paraíba - UFPB, Brasil. E-mail: carlosepimentel@bol.com.br

${ }^{5}$ Doutora em Psicologia Social. Professora da Pós-Graduação em Psicologia Social pela Universidade Federal da Paraíba - UFPB, Brasil. Email: patynfonseca@hotmail.com.

${ }^{6}$ Doutor em Psicologia Social. Professor Adjunto IV do Curso de Graduação em Psicologia e do Programa de Pós-Graduação em Psicologia (Mestrado) da Universidade Federal do Piauí. E-mail: emersondiogenes@gmail.com.

Revista Iberoamericana de Diagnóstico y Evaluación - e Avaliação Psicológica. RIDEP · No55 · Vol.2 · 161-172 2020

ISSN: 1135-3848 print /2183-6051online
} 


\section{Introdução}

$\mathrm{O}$ avanço da tecnologia trouxe um aumento do uso de smartphones, sobretudo na área da comunicação e do trabalho, porquanto é uma ferramenta com recursos avançados que permite ao usuário a navegação na internet, o acesso a aplicativos que ajudam o indivíduo no dia-a-dia (Lin, Griffiths, \& Pakpour, 2018) e o contato pessoal e/ou profissional imediato (Mallya, Kumar, \& Mashal, 2018).

Contudo, o uso indiscriminado do smartphone vem acarretado prejuízos à saúde física e psicológica dos usuários e, consequentemente, a qualidade de vida. Estudos apontam que as pessoas que acessam repetidamente o smartphone podem sofrer de estresse e ansiedade, a ponto de caracterizar uma dependência (Dongre, Inamdar, \& Gattani, 2017; Bragazzi \& Del Puente, 2014).

Assim, a comunidade científica vem buscando conhecer as consequências do uso indevido de novas tecnologias, especialmente aquelas ligadas ao uso de smartphones, sobretudo a dependência que muitos usuários já relatam ter, conhecida como a nomofobia. Este fenômeno é relativamente novo, resultando do desenvolvimento de novas tecnologias de comunicação virtual (Mallya et al., 2018), principalmente os smartphones (Yildirim \& Correia, 2015).

A palavra "nomophobia" tem origem na Inglaterra, derivada da frase "No Mobile Phobia", que se refere ao medo ou fobia patológica de não conseguir se comunicar por meio do smartphone (Dongre et al., 2017; King, Valença, \& Nardi, 2010). Esse fenômeno vem sendo investigado principalmente entre os jovens, população que mais tem consumido este tipo de tecnologia e se deparado com consequências negativas, tais como: afastamento dos relacionamentos presenciais, solidão, ansiedade e baixo nível de bem estar (Bragazzi \& Del Puente, 2013).

Inicialmente, na definição de nomofobia abordava-se apenas algumas sensações, tais como, ansiedade, desconforto, nervosismo e angústia, todos resultantes da indisponibilidade do indivíduo acessar dispositivos de comunicação virtual ou computadores (King et al., 2010; Yildirim \& Correia, 2015). Recentemente o fenômeno foi definido como uma fobia específica, referente a um transtorno de ansiedade e medo irracional causado por um estímulo pormenorizado, como um determinado objeto ou acontecimento (Bragazzi \& Del Puente, 2014).

Essa nova definição traz uma conotação situacional da nomofobia e, portanto, é compreendida como uma ansiedade de separação do smartphone, que leva o usuário a mantê-lo sempre próximo (Han, Kim, \& Kim, 2017). Ademais, o indivíduo apresenta um medo irracional de sair de casa sem o telefone móvel, de perdê-lo, de acabar a bateria ou de ficar sem sinal de internet, o que gera alto nível de ansiedade (Bragazzi \& Del Puente, 2014) e problemas nos relacionamentos sociais, acadêmicos e/ou no trabalho (Gutiérrez-Puertas et al., 2019).

Em uma revisão abrangente da literatura, Bragazzi e Del Puente (2014) atentam para os efeitos nocivos que as novas mídias podem ocasionar, discutindo e sugerindo a inclusão da nomofobia no DSM-V. Entretanto, embora a nomofobia não esteja listada no DSM-V como distúrbio psicológico, pesquisas indicam que o fenômeno pode servir como forte indicador de transtorno ou fobia social para indivíduos com proeminente dependência de se comunicar virtualmente (Han et al., 2017; King, et al., 2013; King et al., 2014).

As pessoas nomofóbicas podem apresentar sintomas de alteração respiratória, transpiração, agitação, desorientação, taquicardia e tremor (Shankar, Singh, \& Jangir, 2018). Também, evitam propositadamente lugares e situações em que o uso de dispositivos móveis é proibido, pois necessitam ficar conectado 24 horas por dia para verificar chamadas e/ou mensagens. Neste caso, percebe-se que o usurário apresenta preferência pela interação virtual em detrimento da real, normalmente tem gastos elevados em função da necessidade de adquirir equipamentos mais sofisticados para se conectar e, por fim, demonstra uma escassez de sono em decorrência do número excessivo de horas que passa navegando pela internet (Bragazzi \& Del Puente, 2014).

Mallaya et al. (2018), em pesquisa realizada com 150 universitários, observaram que a prevalência de nomofobia era em mulheres. Os resultados revelaram que cerca de $87 \%$ dos participantes eram nomofóbicos e $13 \%$ estavam em risco de desenvolvê-la. Além disso, o uso 
frequente do smartphones estava relacionado positivamente com problemas psicológicos e comportamentais, como a compulsividade, aborrecimento, estresse e baixo desempenho acadêmico. Posteriormente, Al-Balhan, Khabbache, Watfa, Zerbetto e Bragazzi (2018) averiguaram, em 512 adolescentes e jovens adultos do Kuwait, uma prevalência de níveis moderado (56.2\%) e grave $(25.8 \%)$ de comportamentos nomofóbicos.

Já no estudo levado a cabo por Durak (2019), com 612 estudantes do ensino fundamental e médio da Turquia, verificou-se uma relação significativa entre vício em smartphones e nomofobia, indicando a presença de sintomas patológicos, a exemplo da depressão, ansiedade, baixa produtividade e rendimento acadêmico. Ademais, foi verificado que os níveis de nomofobia dos alunos aumentaram com a idade. Por esse motivo, a autora aponta que se deve priorizar planos preventivos, para lidar adequadamente com o uso abusivo dos smartphones.

Assim, considerando o explanado até o momento, estima-se ficar claro a importância de se contar com instrumentos de medidas adequados, que permitam avaliar o fenômeno aqui tratado, e conhecer seus antecedentes e consequentes, especialmente entre o público mais atingido pela nomofobia, ou seja, os mais jovens (Gezgin, Cakir, \& Yildirim, 2018; González-Cabrera, León-Mejía, Pérez-Sancho, \& Calvete, 2017). Isto posto, contar com instrumentos psicométricos adequados que auxiliem na compreensão e avaliação do construto, pode contribuir para que os profissionais de saúde possam identificar as comorbidades de transtornos psicológicos relacionados ao fenômeno, uma vez que os diagnósticos preliminares nem sempre são claros (Lee, Kim, Mendoza, \& McDonough, 2018).

Tendo isto em conta, Yildirim e Correia (2015) elaboraram o Nomophobia Questionnaire (NMP-Q), a partir de grupos focais e entrevistas semiestruturadas realizadas com 301 universitários estadunidenses. $\mathrm{O}$ instrumento ficou constituído por 20 itens respondidos em uma escala tipo Likert de 7 pontos, variando de 1 (Discordo totalmente) até 7 (Concordo totalmente).

Por meio da análise dos componentes principais do NMP-Q, identificou-se quatro dimensões que explicavam $69.58 \%$ da variância total. Com base no coeficiente alfa de Cronbach $(\alpha)$, constatou-se a precisão do instrumento para as quatro dimensões, a saber: incapacidade de comunicação $(\alpha=.94)$, perda de conexão $(\alpha=.87)$, incapacidade de acessar informações $(\alpha=.83)$ e renúncia da conveniência $(\alpha=.82)$.

Os autores (Yildirim \& Correia, 2015) descreveram as dimensões como seguem: (a) Incapacidade de comunicação, refere-se a ausência de possibilidade de contatar com outras pessoas. Reunindo sentimentos relacionados a perca da comunicação instantânea com os demais e/ou não ser capaz de utilizar serviços que a promovam; (b) Perda de conexão diz respeito aos sentimentos de perda da conectividade onipresente (sensação de estar presente em todos os lugares simultaneamente) que os smartphones proporcionam, e de ficar desconectado da sua identidade online, principalmente das redes sociais; (c) Incapacidade de acessar informações corresponde ao desconforto generalizado da perda do acesso instantâneo a informações através dos smartphones; e, por fim, (d) Renúncia da conveniência está relacionado a sentimentos de renúncia (abdicação) de convivência com os demais, evidenciados pelas vantagens que o smartphone pode proporcionar, a exemplo de ter acesso constante e instantâneo à internet em qualquer lugar e a qualquer momento. Quando tal conveniência não é suprida, surgem sentimentos de desconforto e ansiedade.

Observa-se que a NMP-Q tem sido amplamente adaptada e utilizada em diferentes países. No contexto espanhol, por exemplo, foram reunidas evidências de qualidade métrica na pesquisa realizada por González-Cabrera et al. (2017), considerando uma amostra de 306 adolescentes, na qual corroborou a solução tetrafatorial e indicou evidências de validade convergente. Na China, Ma e Liu (2018), tendo uma amostra de 966 universitários, indicou a adequação da estrutura proposta originalmente realizando análises exploratória e confirmatória. Ademais, encontrou-se validações transculturais, do NMP-Q, na Itália (Adawi et al., 2018), Irã (Lin et al., 2018), Kwait (Al-Balhan et al., 2018), Paquistão (Nawaz, Sultana, Amjad, \& Shaheen, 2017) e Turquia (Arpaci, Baloğlu, Özteke Kozan, \& Kesici, 2017), dentre outras. Tal fato 
possibilitou averiguar a prevalência e os correlatos da nomofobia em diferentes culturas, além de reunir evidências de validade de construto da medida, considerando diferentes métodos, que atestaram a robustez da estrutura da NMP-Q.

Apesar da ampla utilização da NMP-Q em diferentes culturas, não foram encontrados estudos com amostras brasileiras, nem tampouco adaptações para o português do Brasil, evidenciando-se, portanto, uma lacuna no cenário nacional, fato que justifica os esforços para lograr o objetivo principal aqui proposto, que é o de adaptar o Nomophobia Questionnaire (Yildirim \& Correia 2015) para o português brasileiro, reunindo evidências de validade e precisão da medida. Com este intuito levou-se a cabo dois estudos empíricos independentes, que são apresentados a seguir.

\section{Método}

Estudo 1. Adaptação e evidências de validade e precisão da Nomophobia Questionnaire.

\section{Participantes}

Contou-se com uma amostra não probabilística (por conveniência), que totalizou 219 respondentes de vários estados brasileiros, sendo a maioria da Paraíba (75.6\%). Estes possuíam idades que variaram de 18 a 58 anos $(M=27.89 ; D P=7.99)$, sendo a maioria do sexo feminino (69.1\%), solteiros (70.9\%) e com ensino superior incompleto (36\%), que relataram passar em média 5.7 horas diárias $(D P=1.07$; variando de 1 a 20) usando o smartphone.

\section{Instrumentos}

Os participantes responderam a um livreto contendo os seguintes instrumentos:

Nomophobia Questionnaire (NMP-Q; anexo 1). Trata-se de um instrumento elaborado por Yildirim e Correia (2015), composto por 20 itens respondidos em uma escala tipo Likert de 7 pontos, que variam desde 1 (Discordo totalmente) até 7 (Concordo totalmente). O conjunto de 20 itens são distribuídos entre as quatro dimensões: (1) Incapacidade de comunicação (2) Perda de conexão (3); Incapacidade de acessar informações (4) Renúncia da conveniência.
Questionário sociodemográfico: conjunto de perguntas que tinham objetivo de caracterizar a amostra, a exemplo das seguintes variáveis: idade, sexo, estado civil, nível de escolaridade e tempo de uso diário do smartphone.

\section{Procedimento}

Inicialmente, procedeu-se a adaptação NMP-Q para o português brasileiro, sendo utilizada a técnica back translation. Assim, um pesquisador bilíngue, realizou a tradução do inglês para o português brasileiro. Posteriormente, a versão inicial em português, foi retraduzida para o idioma original (inglês estadunidense), por um segundo pesquisador independente, para que fossem observadas equivalências dos itens das duas versões. Com a equivalência garantida da versão preliminar em língua brasileira, foi realizada a validação semântica, como sugerido por Pasquali (2016), buscando avaliar se os itens e o formato da medida estavam compreensíveis. Para tanto, foram considerados 30 pessoas da população geral da cidade de João Pessoa, com níveis de escolaridade entre ensino fundamental a superior. Não havendo necessidade de modificações, partiu-se para a coleta dos dados. A versão adaptada para o português brasileiro pode ser consultada no Anexo 1 .

A coleta deu-se em formato eletrônico, através do Google Docs, disponibilizado aos participantes através de um link que foi divulgado em redes sociais (por exemplo, Facebook, Instagram e Twitter) e via e-mail. $\mathrm{Na}$ oportunidade, foi considerada a técnica de bola de neve (Vinuto, 2014), na qual um participante indicava e/ou convidava seus contatos para participarem da pesquisa. Aos que aceitaram colaborar com a pesquisa, eram esclarecidos os propósitos do estudo, além de serem assegurados o anonimato e o sigilo. Foi destacado que a participação se daria voluntariamente e não traria ônus ou bônus diretos, podendo os mesmos desistir a qualquer momento. Ressalta-se que todos os procedimentos utilizados nesta pesquisa foram respeitaram as recomendações éticas para pesquisas com seres humanos conforme as Resoluções 510/16 e 466/12 do Conselho Nacional de Saúde. Os participantes precisaram de 10 a 15 minutos, em média, para participarem da pesquisa. 
Quadro 1. Estrutura Fatorial do Nomophobia Questionnaire

\begin{tabular}{|c|c|c|c|c|c|}
\hline Fatores/ Itens & F1 & $\mathrm{F} 2$ & F3 & $\mathrm{F} 4$ & $\mathrm{~h}^{2}$ \\
\hline \multicolumn{6}{|c|}{ Fator 1 Incapacidade de comunicação } \\
\hline Item 13. & $.99 *$ & -.13 & .01 & .02 & .87 \\
\hline Item 11. & $.90 *$ & -.11 & -.01 & -.06 & .66 \\
\hline Item 10. & $.85^{*}$ & .07 & -.04 & -.04 & .72 \\
\hline Item 15. & $.70 *$ & .17 & .04 & .08 & .79 \\
\hline Item 14. & $.57 *$ & .17 & -.03 & .23 & .70 \\
\hline Item 12. & $.52 *$ & .06 & .04 & .27 & .61 \\
\hline \multicolumn{6}{|l|}{ Fator $2=$ Perda de conexão } \\
\hline Item 18. & 01 & $.98^{*}$ & .02 & -.17 & .81 \\
\hline Item 17. & -.17 & $.94 *$ & .06 & .02 & .80 \\
\hline Item 16. & -.01 & $.72 *$ & -.01 & .13 & .65 \\
\hline Item 19. & .10 & $.59 *$ & -.10 & .10 & .45 \\
\hline Item 20. & .07 & $.57 *$ & -.15 & .22 & .49 \\
\hline Item 09. & .40 & .43 & .18 & -.29 & .49 \\
\hline Item 07. & .16 & .29 & .26 & .12 & .47 \\
\hline \multicolumn{6}{|c|}{ Fator 3 = Incapacidade de acessar informações } \\
\hline Item 04. & .12 & -.15 & $.84 *$ & .01 & .68 \\
\hline Item 02. & -.03 & -.03 & $.82 *$ & .02 & .63 \\
\hline Item 01. & -.04 & -.07 & $.67 *$ & -.07 & .49 \\
\hline Item 03. & -.24 & .19 & $.47 *$ & .30 & .48 \\
\hline \multicolumn{6}{|l|}{ Fator 4 = Renúncia da conveniência } \\
\hline Item 05. & .05 & -.03 & .04 & $.77 *$ & .65 \\
\hline Item 06. & -.07 & .09 & .03 & $.70 *$ & .54 \\
\hline Item 08. & .29 & -.09 & -.05 & $.51 *$ & .41 \\
\hline Número de itens & 6 & 5 & 4 & 3 & \\
\hline Valor próprio & 9.55 & 1.86 & 1.31 & 1.14 & \\
\hline Variância explicada (\%) & 47.75 & 9.32 & 6.56 & 5.69 & \\
\hline Alfa de Cronbach & .93 & .88 & .82 & .76 & \\
\hline
\end{tabular}

Nota. * Item retido no fator.

\section{Análise de dados}

Os dados foram analisados através do pacote estatístico SPSS em sua versão 21. Realizaram-se estatísticas descritivas (medidas de tendência central e dispersão), e multivariada (análise fatorial exploratória, com extração dos Eixos Principais), considerando a rotação promax. Optou-se por esse tipo de rotação, por ser mais adequada em ciências psicológicas, pois trata-se de um método oblíquo que não delimita, a priori, interação entre os fatores (Damásio, 2012). Além de calcular os índices de consistência interna (alfa de Cronbach).

\section{Resultados}

Para se verificar a estrutura fatorial, optou-se por realizar uma análise fatorial exploratória (AFE), com o método de extração de eixos principais. Para tanto, buscou-se verificar se os dados são passíveis de fatoração, através do Kaiser-Meyer-Olkin (KMO) e o teste de esfericidade de Bartlett, os resultados encontrados apoiam a realização da análise fatorial, são eles: $\mathrm{KMO}=.93$ e $\chi^{2} \quad(190)=2991.10 ; p<.001$. Inicialmente, realizou-se essa análise sem fixar o número de fatores a serem extraídos, e adotando rotação promax. Na oportunidade, foi possível identificar a extração de quatro fatores com valores próprios (eigenvalue) superior a 1, que explicaram $51,46 \%$ da variância total, solução apoiada pela observância do scree plot (critério de Cattel).

Adotou-se valores iguais ou superiores a $|0,47|$ para reter o item no fator. Assim, os itens 07 [Se não conseguisse me conectar ao Wi-Fi ou não tivesse um sinal de dados móveis (4G, $3 G$, etc), eu ficaria constantemente verificando se havia um sinal ou tentaria encontrar uma rede Wi-Fi.] e 09 [Se ficasse um tempo sem poder verificar meu smartphone, sentiria o desejo (ou vontade) de verificar se tenho novas notificações.] foram eliminados por não obedecerem ao critério adotado. Dessa forma, os 18 itens compuseram a versão em português brasileiro, com cargas fatoriais e alfas de Cronbach variando de .47 a .99 e de .76 a 96 , respectivamente. Os resultados são sumarizados na Quadro 1.

Considerando os resultados apresentados na Quadro 1. Pode-se observar evidências que atestam as qualidades métricas do Nomophobia Questionnaire (NMP-Q), ou seja, evidências de validade e precisão do instrumento na amostra 
considerada. Entretanto, faz-se necessário reconhecer a natureza essencialmente exploratória da técnica estatística empregada no presente estudo. Assim, ressalta-se a emergência de serem realizadas pesquisas visando comprovar a estrutura fatorial da NMP-Q. Desta forma, foi realizado um segundo estudo objetivando comprovar a estrutura deste instrumento, com análises mais robustas, que será apresentado na sequência.

\section{Estudo 02- Comprovação da estrutura fatorial do Nomophobia Questionnaire (NMP-Q)}

\section{Participantes}

Participaram 277 pessoas da população geral de vários estados brasileiros, recrutados de forma não probabilística (acidental). Estes possuíam idades variando de 18 a 58 anos $(M=27.89$; $D P=7.99)$, sendo em sua maioria do Estado da Paraíba (36.1\%), do sexo feminino (66.1\%), solteiros (74\%), com ensino superior incompleto (39\%), os quais relataram passar em média 5,7 horas diárias $(D P=3.89$; variando de 1 a 19$)$ usando o smartphone.

\section{Instrumentos}

Os participantes responderam os seguintes instrumentos: Nomophobia Questionnaire (NMPQ; anexo 1) e perguntas demográficas, descritos detalhadamente no Estudo 1. Ademais, foi incluído short version of the Smartphone Addiction Scale (SAS) elaborado por Kwon, Kin, Cho e Yang (2013) e adaptado para o contexto brasileiro por Mescollotto, Castro, Pelai, Pertille e Bigaton (2018), com precisão assegurada $(\alpha=.95)$. Trata-se de uma medida composta por dez itens, que avaliam de forma global o vício no smartphone, respondidos numa escala do tipo likert variando de 1 (discordo totalmente) a 6 (concordo totalmente). $\mathrm{Na}$ presente pesquisa a confiabilidade foi adequada $(\alpha=.89)$.

\section{Procedimento}

Adotou-se os mesmos procedimentos descritos no Estudo 1 e obedecidas as orientações previstas nas Resoluções 466/12 e 510/16 do Conselho Nacional de Saúde.

\section{Análise da dados}

Os dados foram tabulados e analisados nos pacotes estatísticos SPSS e AMOS, ambos versão
21. Com o primeiro, foram calculadas estatísticas descritivas (medidas de tendência central e dispersão), correlações de Pearson e consistência interna (alfa de Cronbach). Com o AMOS, foi realizada uma Análise Fatorial Confirmatória (AFC), considerando os seguintes indicadores de ajuste (Hair, Black, Babin, Anderson, \& Tatham, 2009; Marôco, 2014; Tabachnick, \& Fidell, 2013): (1) $\chi^{2} / g l$ (razão entre o $\chi^{2}$ e graus de liberdade), com ajustamento podendo ser considerado perfeito $\left(1.0<\chi^{2} / g l<3\right)$, aceitável $\left(3<\chi^{2} / g l<5\right)$ e inaceitáveis $\left(\chi^{2} / g l>5\right)$ e o (2) Comparative Fit Index (CFI) admitindo-se, em ambos, valores a partir de .90, como referências de um modelo ajustado; (3) Tucker-Lewis Index (TLI) sendo aceitável coeficiente acima de .90; (4) Root-Mean-Square Error of Approximation (RMSEA) com valores inferiores a .06 indicando ajuste satisfatório, admitindo-se até .10.

Finalmente, visando reunir evidências de precisão da $N M P-Q$, calculou-se o alfa de Cronbach e a confiabilidade composta (CC). Em ambos os indicadores, os valores iguais ou superiores a .70 asseguram a consistência interna. A CC é um indicador adicional de precisão, com vantagem de não ser influenciado pela quantidade de itens (Marôco, 2014).

\section{Resultados}

Realizou-se uma AFC, com estimador $M L$ (MaximumLikelihood). Os resultados da SEM (Structural Equation Modeling) apresentaram indicadores de adequação de ajuste considerados aceitáveis: $\chi^{2}(129)=353.01, p<.001 ; \chi^{2} / \mathrm{gl}=2.74$, $\mathrm{CFI}=.94, \quad \mathrm{TLI}=.93$, RMSEA $=.08 \quad(\mathrm{IC} 90 \%=.07-$ $.09)$, ressalta-se que todas as cargas fatoriais foram estatisticamente diferentes de zero $(\lambda \neq 0$; $\mathrm{z}>1.96, p<.05)$.

Com a estrutura tetrafatorial assegurada, averiguou-se a consistência interna ( $\alpha$ ) e CC dos fatores do Nomophobia Questionnaire (NMP-Q), que se apresentaram todos como satisfatórios, a saber: Incapacidade de comunicação $(\alpha=.95$ e $\mathrm{CC}=.94)$, Perda de conexão $(\alpha=.90$ e $\mathrm{CC}=.90)$, Incapacidade de acessar informações $(\alpha=.71 \mathrm{e}$ $\mathrm{CC}=.72)$ e, por fim, Renúncia da conveniência $(\alpha=.84$ e $\mathrm{CC}=.84)$. Ainda com o objetivo de reunir evidências complementares de validade, buscou-se 


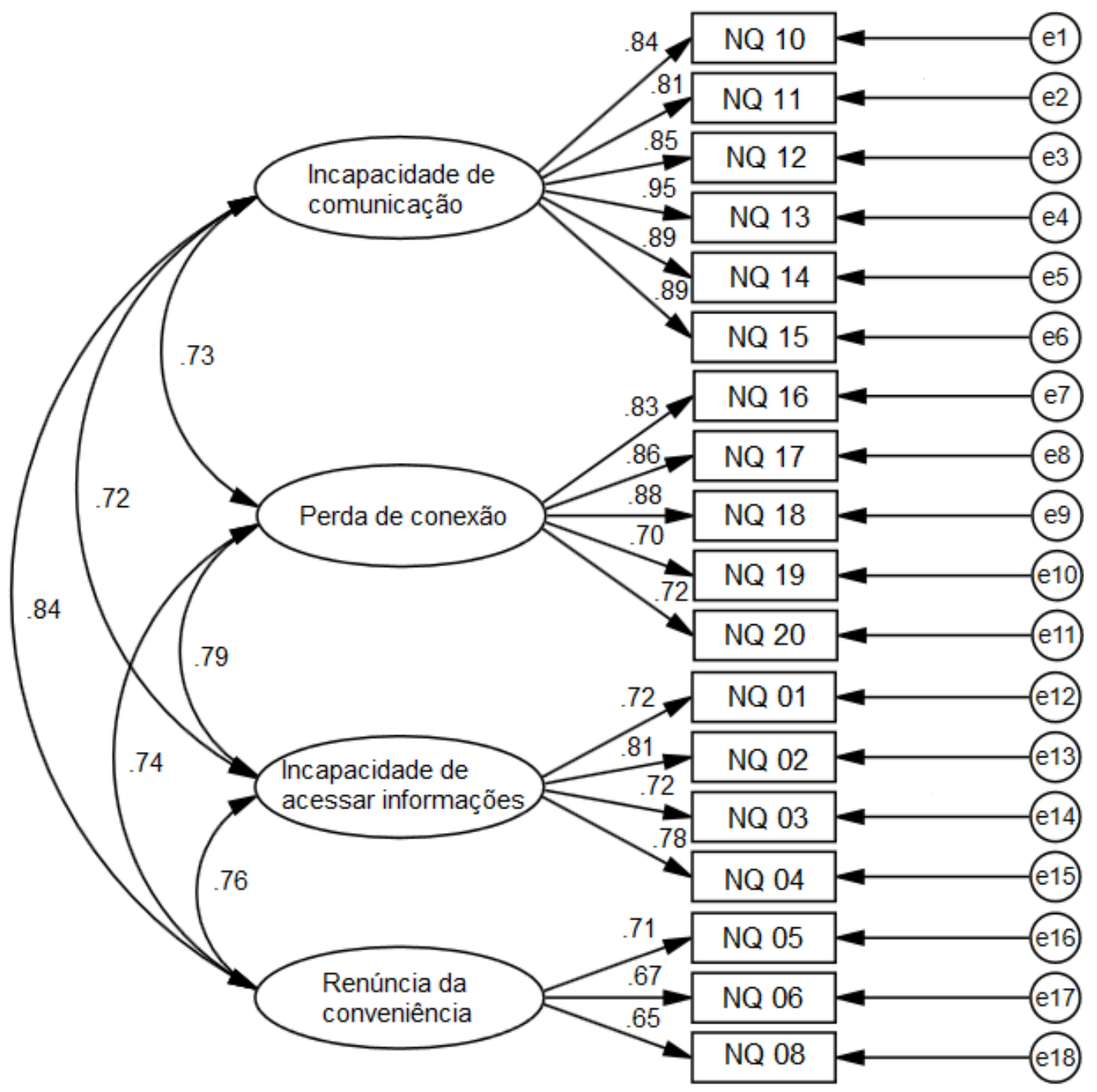

Figura 1. Estrutura Fatorial do Nomophobia Questionnaire

conhecer a associação entre os fatores do NMP-Q e da SAS. Os resultados indicaram relação positiva da pontuação total da SAS com incapacidade de comunicação $(\mathrm{r}=.61 ; p<.001)$, perda de conexão $(\mathrm{r}=.71 ; p<.001)$, incapacidade de acessar informações $(\mathrm{r}=.60 ; p<.001)$ e renúncia da conveniência $\left(\mathrm{r}=.61 ; p^{<.001)}\right.$, podendo ser interpretados como indicadores de validade convergente.

\section{Discussão}

Considerando a nomofobia, como um construto relativamente novo, aliado a escassez de pesquisas no contexto brasileiro, decidiu-se empreender esforços para contribuir com o tema, procurando incialmente adaptar a Nomophobia Questionnaire (NMP-Q; Yildirim \& Correia, 2015), reunindo evidências de sua validade (fatorial e convergente) e consistência interna com amostras brasileiras. Estima-se que os objetivos tenham sido alcançados, como pode ser visualizado a partir dos dois estudos independente, que evidenciam parâmetros psicométricos meritórios para a NMP-Q. Deste modo, a seguir, os principais resultados serão discutidos.

Inicialmente, destaca-se que a estrutura tetrafatorial original foi atestada empiricamente no Estudo 1, por meio de análise fatorial exploratória, com rotação promax. A versão brasileira ratifica achados de pesquisas transculturais prévias (Adawi et al., 2018; Lin et al. 2018; Gutiérrez-Puertas et al. 2019; Nawaz et al., 2017), apresentando-se uma versão da NMPQ, com menor número de itens, composta por 18 itens. Os itens 7 e 9 foram excluídos da versão final, adaptada ao contexto nacional, pois apresentaram saturação considerável em fator contraditório ao originalmente proposto por seus autores (Yildirim \& Correia, 2015). 
Nessa direção, apesar de em algumas culturas serem mantidas a estrutura e o conjunto de itens originais (e.g. Adawi et al., 2018; Yildirim \& Correia, 2015; Gutiérrez-Puertas et al., 2019), em algumas outras os itens apresentaram problemas, ocasionando a sua exclusão. Por exemplo, na versão Persa optou-se por excluir os itens 9 e 14, por apresentarem baixas cargas fatoriais em seus fatores teóricos (Lin et al., 2018). De maneira similar, a versão Árabe, especificamente no Kwait (Al-Balhan et al., 2018), os itens 03 e 17 apresentaram inconsistências entre sua carga fatorial encontrada e sua alocação no fator teoricamente esperado. Possivelmente, isto é explicado em função das diferenças culturais ou amostrais (Ma \& Liu, 2018).

Posteriormente, por meio da AFC, no Estudo 2, foi possível encontrar indicadores de ajustes que endossaram à estrutura encontrada no estudo prévio (18 itens distribuídos por 4 dimensões). O modelo tetrafatorial apresentou bom ajuste aos dados, (CFI=.94, TLI=.93, RMSEA=.08), em consonância com o recomendado na literatura (Hair et al., 2009), isto é, CFI e TLI>.90 e RMSEA=.08). Para além disso, foram reunidas evidências complementares de validade de construto, por meio da validade convergente (Marôco, 2014). Para tanto, utilizou-se da SAS (Kwon et al., 2013), que avalia o vício no smartphone, estima-se, teoricamente, que os fatores da NMP-Q se relacionassem positivamente com as pontuações da SAS (Nawaz et al., 2017). Os resultados desta análise revelaram forte associação $(r>.60$ e $p<.05)$ entre os fatores das escalas, como esperado. $\mathrm{O}$ que pode ser considerado evidências adicionais de validade convergente da NMP-Q, a semelhança com estudos prévios (Gezgin et al., 2018; Ma \& Liu, 2018) que indicavam tal associação. Nessa direção, Yildirim e Correia (2015) argumentam que o uso dos smartphones tende a aumentar a gravidade dos sintomas de nomofobia, devido aos equipamentos eletrônicos apresentarem cada dia mais recursos (e.g., acesso à internet, redes socias, notificações instantâneas, entre outras funções), ocasionando sentimentos mais intensos de ansiedade e angústia, quando os mesmos não conseguem acessá-lo.

No que concerne a consistência interna e confiabilidade composta (CC), estima-se que estas podem ser consideradas aceitáveis, de acordo com as recomendações da literatura, ressaltando assim, que os valores para os dois indicadores foram superiores a .70, ficando atestado evidências deste parâmetro (Morôco, 2014; Pasquali, 2016). Especificamente, na presente pesquisa, observa-se que a precisão, avaliada através do $\alpha$ (alfa de Cronbach), variou entre .70 a .93 (Estudo 1) e .72 a .95 (Estudo 2) considerando todos os quatro fatores em ambos os estudos. Esses valores são similares a estudos prévios realizados em outras culturas: .81 a .95 (Yildirim \& Correia, 2015) e .79 a .92 (González-Cabrera et al., 2017).

Apesar dos resultados favoráveis, tem-se em conta que esta pesquisa não se encontra isenta de potenciais limitações. Dito isto, cita-se inicialmente a amostra, que foi angariada de maneira conveniente (não probabilística), por meio da técnica bola de neve, que apesar de possibilitar um alcance maior, não proporciona uma amostra representativa da população (Brabete, 2014), contando apenas com a colaboração de participantes que, convidados, aceitaram responder a pesquisa e indicaram outros participantes por intermédio das redes sociais ou aplicativos de mensagens, impossibilitando um controle maior de participantes e conseguinte generalização dos resultados para além da amostras consideradas na pesquisa. Desta forma, ressalta-se que não se objetivou generalizações, mas apresentar uma medida sobre nomofobia que reunisse evidências de validade e precisão.

Outra possível limitação refere-se ao fato de os dados serem coletados por uma medida de autorrelato, pois existe uma indicação de que isto pode enfraquecer a validade da medida (Lin et al., 2018), pois considerando que a nomofobia não é um comportamento socialmente desejável, fato que possibilita que as pessoas falseiem suas respostas visando promoção pessoal (Gouveia, Guerra, Souza, Santos, \& Costa, 2009).

Ademais, mesmo considerando as potenciais limitações supracitadas, acredita-se que os mesmos tenham sido logrados com êxito, pois deve-se ter em conta a ênfase psicometrista dos estudos aqui apresentados, que procurou adaptar para o português brasileiro, além de reunir evidências de validade e precisão de uma medida de nomofobia.

Para estudos futuros, sugere-se amostras mais 
heterogêneas que considerem participantes das cinco regiões brasileiras, além de pessoas com distintas fases do desenvolvimento humano, possibilitando averiguar, por exemplo, em qual faixa etária da população brasileira existe uma maior prevalência de comportamentos nomofóbicos, uma vez que a idade tem se demonstrado como importante preditor desta conduta, percebida em sua maioria em adolescentes e jovens adultos (Al-Balhan et al., 2018; Ma \& Liu, 2018).

Além disso, faz-se necessário que pesquisas futuras ampliem os contextos de investigação, averiguando evidências adicionais de validade, tais como a convergente-discriminante e de critério, além da invariância fatorial em diferentes grupos, a exemplo de adolescentes e jovens adultos, que são considerados mais vulneráveis a prevalência da nomofobia (González-Cabrera et al., 2017), além de verificar possíveis diferenças em função do sexo ou checar a estabilidade temporal (teste-reteste) do instrumento.

Em suma, os resultados oriundos dos dois estudos endossam a estrutura tetrafatorial da medida (Gezgin et al., 2018; Nawaz et al., 2017; Yildirim \& Correia, 2015), reunindo evidências psicométricas (validade) que justificam seu emprego em pesquisas futuras. A medida também apresentou índices de precisão que variam de razoáveis a excelentes. Assim, a NMP-Q apresenta-se como uma fermenta adequada, que pode ser útil para conhecer antecedentes $\mathrm{e}$ consequentes associados com a nomofobia. Possibilitando, portanto, entender os efeitos negativos atribuídos ao fenômeno (Arpaci et al., 2017; Bragazzi \& Del Puente, 2014; Yildirim, Degen, Tanenhaus, \& Jaeger, 2016).

Por fim, acredita-se que a presente pesquisa pode ser um dos primeiros passos para que haja avanços teóricos e metodológicos para o entendimento da nomofobia no Brasil. Assim, espera-se que o estudo auxilie na elaboração e implementação de estratégias para combater os efeitos nocivos do uso inadequado das novas tecnologias, que possibilitou uma maior interação com a presencia de Internet, fazendo-se necessário avaliar a prevalência e os processos que mantem essas patologias e outros processos que afetam de maneira significativa a qualidade de vida (Contreras \& Ottenberger, 2017), em especial o smartphone.

\section{Referências}

Arpaci, I., Baloğlu, M., Özteke Kozan, H. İ., \& Kesici, Ş. (2017). Individual differences in the relationship between attachment and nomophobia among college students: The mediating role of mindfulness. Journal of medical Internet research, 19(12), e404. doi:10.2196/jmir.8847

Adawi, M., Bragazzi, N. L., Argumosa-Villar, L., Boada-Grau, J., Vigil-Colet, A., Yildirim, C., ... Watad, A. (2018). Translation and validation of the nomophobia questionnaire in the italian language: Exploratory factor analysis. JMIR Mhealth And Uhealth, 6(1), e24. doi:10.2196/preprints.9186.

Al-Balhan, E. M., Khabbache, H., Watfa, A., Re, T. S., Zerbetto, R., \& Bragazzi, N. L. (2018). Psychometric evaluation of the Arabic version of the nomophobia questionnaire: Confirmatory and exploratory factor analysis - implications from a pilot study in Kuwait among university students. Psychology Research and Behavior Management, 11, 471-482. doi:10.2147/prbm.s169918

Brabete, A. C. (2014). El Cuestionario de Salud General de 12 items (GHQ12): Estudio de traducción y adaptación de la versión rumana. Revista Iberoamericana de Diagnóstico y Evaluación - e Avaliação Psicológica, 37(1), 11-29. Recuperado de https://www.aidep.org/sites/default/files/201812/Art1.pdf

Bragazzi N. L., \& Del Puente G. (2014). A proposal for including nomophobia in the new DSM-V. doi:10.2147/PRBM.S41386

Contreras, C. H., \& Ottenberger, D. R. (2018). Adaptación transcultural y evaluación de las estructuras factoriales del test de adicción a internet en Chile: Desarrollo de una Versión Abreviada. Revista Iberoamericana de Diagnóstico y Evaluación - e Avaliação Psicológica, 49(4), 143-155. doi:10.21865/RIDEP49.4.12

Damásio, B. (2012). Uso da análise fatorial exploratória em psicologia. Avaliação Psicológica, 11(2), 213-228. Recuperado de http://pepsic.bvsalud.org/pdf/avp/v11n2/v11n 2a07.pdf 
Dongre, A. S., Inamdar. I. F., \& Gattani, P., L. (2017). Nomophobia: A Study to Evaluate Mobile Phone Dependence and Impact of Cell Phone on Health. National Journal of Community Medicine, 8(11), 688-693.

Durak, H. Y. (2019). Investigation of nomophobia and smartphone addiction predictors among adolescents in Turkey: Demographic variables and academic performance. The Social Science Journal, 56(4), 492-517. doi:10.1016/j.soscij.2018.09.003

Gezgin, D, Cakir, O., \& Yildirim, S. (2018). The relationship between levels of nomophobia prevalence and internet addiction among high school students: The factors influencing Nomophobia. International Journal of Research in Education and Science, 4(1), 215225. doi:10.21890/ijres.383153

González-Cabrera, J., León-Mejía, A., PérezSancho, C., \& Calvete, E. (2017). Adaptation of the Nomophobia Questionnaire (NMP-Q) to Spanish in a sample of adolescents. Actas Espanolas de Psiquiatría, 45(4), 137-144. Recuperado de https://www.ncbi.nlm.nih.gov/pubmed/28745 386

Gouveia, V. V., Guerra, V. M., Sousa, D. M. F., Santos, W. S., \& Costa, J. M. (2009). Escala de Desejabilidade Social de MarloweCrowne: Evidências de sua validade fatorial e consistência interna. Avaliação Psicológica, 8(1), 87-98. Recuperado de http://pepsic.bvsalud.org/scielo.php?script=sci _arttext\&pid=S1677$04712009000100008 \& \operatorname{lng}=\mathrm{pt} \& \operatorname{tlng}=\mathrm{pt}$.

Gutiérrez-Puertas, L., Márquez-Hernández, V. V., São-Romão-Preto, L., Granados Gámez, G., Vanesa Gutiérrez-Puertas V., \& AguileraManrique, G. (2018). Comparative study of nomophobia among Spanish and Portuguese university students. Nomophobia in nursing students, Nurse Education in Practice, 34, 7984. doi:10.1016/j.nepr.2018.11.010

Hair, J. F., Jr., Black, W. C., Babin, B. J., Anderson, R. E., \& Tatham, R. L. (2009). Análise multivariada de dados. (6a ed.) Porto Alegre, RS: Bookman.

Han, S., Kim, K. J., Kim, J. H. (2017). Understanding nomophobia: Structural Equation modeling and semantic network analysis of smartphone separation anxiety. Cyberpsychology, Behavior, and Social Networking, 20(7), 419-427 doi:10.1089/cyber.2017.0113

Kanmani A., Bhavani U., \& Maragatham R. (2017). Nomophobia - an insight into its psychological aspects in India. The International Journal of Indian Psychology, 4(2), 1-15. doi:10.25215/0402.041

King, A. L. S., Valença, A. M., \& Nardi, A. E. (2010). Nomophobia: The mobile phone in panic disorder with agoraphobia: Reducing phobias or worsening of dependence? Cognitive and Behavioral Neurology, 23(1), 52-54. doi:10.1097/WNN.0b013e3181b7eabc.

King, A. L. S., Valença, A. M., Silva, A. C. O., Baczynski, T., Carvalho, M. R., \& Nardi, A. E. (2013). Nomophobia: Dependency on virtual environments or social phobia? Computers in Human Behavior, 29(1), 140144. doi:10.1016/j.chb.2012.07.025

King, A. L., Valença, A. M., Silva, A. C., Sancassiani, F., Machado, S., \& Nardi, A. E. (2014). "Nomophobia": Impact of cell phone use interfering with symptoms and emotions of individuals with panic disorder compared with a control group. Clinical Practice and Epidemiology in Mental Health, 10, 28-35. doi:10.2174/1745017901410010028

Kwon, M., Kim, D. J., Cho, H., \& Yang, S. (2013). The smartphone addiction scale: Development and validation of a short version for adolescents. PLoS One, 8(12), e83558. doi:10.1371/journal.pone.0083558.

Lee, S., Kim, M., Mendoza, J. S., \& McDonough, I. M. (2018). Addicted to cellphones: exploring the psychometric properties between the nomophobia questionnaire and obsessiveness in college students. Heliyon, 4(11), e00895. doi:10.1016/j.heliyon.2018.e00895

Lin, C. Y., Griffiths, M. D., \& Pakpour, A. H. (2018). Psychometric evaluation of Persian Nomophobia Questionnaire: Differential item functioning and measurement invariance across gender. Journal of behavioral addictions, 7(1), 100-108. doi:10.1556/2006.7.2018.11

Ma, J., \& Liu, C. (2018). Evaluation of the factor structure of the Chinese version of the 
nomophobia questionnaire. Current Psychology. doi:10.1007/s12144-018-0071-9

Mallya, N. V., Kumar, S. D. R., \& Mashal, S. (2018) A study to evaluate the behavioral dimensions of "nomophobia" and attitude toward smartphone usage among medical students in Bengaluru. National Journal of Physiology, Pharmacy and Pharmacology, $8(11)$, 1553-1557. doi:10.5455/njppp.2018.8.0827603092018

Marôco, J. (2014). Análise de equações estruturais: Fundamentos teóricos, software e aplicações (2.ed.). Pêro Pinheiro, Portugal: Report Number.

Mescollotto, F. F., Castro, E. M., Pelai, E. B., Pertille, A., \& Bigaton, D. R. (2018). Translation of the short version of the Smartphone Addiction Scale into Brazilian Portuguese: Cross-cultural adaptation and testing of measurement properties. Brazilian Journal of Physical Therapy, 22(4), 276-282. doi:10.1016/j.bjpt.2018.08.013

Nawaz, I., Sultana, I., Amjad, M. J., \& Shaheen, A. (2017). Measuring the enormity of nomophobia among youth in Pakistan. Journal of Technology in Behavioral Science, 2(3-4), 149-155. doi:10.1007/s41347-017-0028-0

Pasquali, L. (2016). TEP - Técnicas de Exame Psicológico: os fundamentos. ( $2^{\mathrm{a}}$ ed). São Paulo, SP: Vetor editora.

Rangka, I. B., Prasetyaningtyas, W. E., I Ifdil, I., Ardi, Z., Suranata, K, Winingsih, E., Sofyan, A., Irawan, M., Arjanto, P., Muslifar, R., \& Wijaya, R. S. (2018). Measuring psychometric properties of the Indonesian version of the NoMoPhobia Questionnaire (NMPQ): Insight from Rasch measurement tool. Journal of Physics: Conference Series, doi:10.1088/1742-6596/1114/1/01212745678

Shankar, V., Singh, K., \& Jangir, M. K. (2018). NOMOPHOBIA: Detection and analysis of smartphone addiction in Indian perspective. International Journal of Applied Engineering Research, 13(14), 11593-11599. Recuperado de

https://pdfs.semanticscholar.org/fdc9/00cff6e5 b8f97fe8d6f5c342b81031bc4ff5.pdf?_ga=2.1

24071306.1026290482.1580419679-

1775492842.1561129897
Tabachnick, B. G., \& Fidell, L. S. (2013). Using multivariate statistics (6th ed.) New Jersey: Pearson Education.

Vinuto, J. (2014). A amostragem em bola de neve na pesquisa qualitativa: Um debate em aberto. Temáticas (Campinas), 22(44), 203-220. Recuperado de https://www.ifch.unicamp.br/ojs/index.php/te maticas/article/view/2144/1637

Yildirim, C., \& Correia, A.-P. (2015). Exploring the dimensions of nomophobia: Development and validation of a self-reported questionnaire. Computers in Human Behavior, 49, 130-137.

doi: doi.org/10.1016/j.chb.2015.02.059

Yildirim, I., Degen, J., Tanenhaus, M. K., \& Jaeger, T. F. (2016). Talker-specificity and adaptation in quantifier interpretation. Journal of memory and language, 87, 128-143. doi:10.1016/j.jml.2015.08.003 


\section{Anexo 1.}

Nomophobia Questionnaire: versão adaptada para o contexto brasileiro

INSTRUÇÕES: Por favor, indique o grau de concordância ou discordância em relação as declarações abaixo, que são referentes aos seus hábitos de uso do seu smartphone.

\begin{tabular}{|c|c|c|c|c|c|c|}
\hline 1 & 2 & 3 & 4 & 5 & 6 & 7 \\
\hline $\begin{array}{c}\text { Discordo } \\
\text { totalmente }\end{array}$ & Discordo & $\begin{array}{c}\text { Discordo } \\
\text { parcialmente }\end{array}$ & $\begin{array}{c}\text { Nem } \\
\text { concordo } \\
\text { nem } \\
\text { discordo }\end{array}$ & $\begin{array}{c}\text { Concordo } \\
\text { parcialmente }\end{array}$ & Concordo & $\begin{array}{c}\text { Concordo } \\
\text { totalmente }\end{array}$ \\
\hline
\end{tabular}

1. Sentirme-ia desconfortável, caso não tivesse acesso constante a informações através do meu smartphone.

2. Eu ficaria chateado se não pudesse buscar informações no meu smartphone quando sentisse vontade.

3. Não conseguir receber notícias (por exemplo, acontecimentos, previsão do tempo, etc.) no meu smartphone me deixaria nervoso(a).

4. Ficaria chateado se eu não pudesse usar o meu smartphone e/ou suas funcionalidades quando sentisse vontade.

5. Ficar sem bateria no meu smartphone iria me assustar.

6. Entraria em pânico se ficasse sem créditos ou atingisse meu limite de dados mensais..

7. Se não conseguisse me conectar ao Wi-Fi ou não tivesse um sinal de dados móveis (4G, 3G, etc), eu ficaria constantemente verificando se havia um sinal ou tentaria encontrar uma rede Wi-Fi.

8. Teria medo de ficar preso em algum lugar, caso o meu smartphone não funcionasse ou eu não pudesse usá-lo.

9. Se ficasse um tempo sem poder verificar meu smartphone, sentiria o desejo (ou vontade) de verificar se tenho novas notificações.

\section{Se meu smartphone não estivesse comigo...,}

10. Sentir-me-ia ansioso pois não conseguiria me comunicar instantaneamente com minha família e/ou amigos.

11. Ficaria preocupado porque minha família e/ou amigos não poderiam entrar em contato comigo.

12. Ficaria nervoso porque não seria capaz de receber mensagens de texto e chamadas.

13. Ficaria ansioso porque não conseguiria manter contato com minha família e/ou amigos.

14. Ficaria nervoso porque não poderia saber se alguém tentou se comunicar comigo.

15. Sentiria-me ansioso porque minha conexão constante com minha família e amigos seria interrompida.

16. Ficaria nervoso porque estaria desconectado da minha identidade virtual (online).

17. Ficaria desconfortável porque eu não poderia manter-me a par (ficar em dia) do que ocorre nas redes sociais e em outros meios de comunicação online.

18. Sentiria-me desconfortável porque não poderia checar minhas notificações de atualizações de minhas mensagens e redes sociais.

19. sentiria-me ansioso(a) porque não podia checar minhas mensagens de e-mail

20. Sentiria-me icomodado(a) e estranho porque não saberia o que fazer. 